Rabaska

Revue d'ethnologie de l'Amérique française

\title{
L'édition des contes de tradition orale. Pour qui ? Comment ?
}

\section{Jean-Pierre Pichette et Carmen d'Entremont}

Volume 7, 2009

URI : https://id.erudit.org/iderudit/038345ar

DOI : https://doi.org/10.7202/038345ar

Aller au sommaire du numéro

Éditeur(s)

Société québécoise d'ethnologie

ISSN

1703-7433 (imprimé)

1916-7350 (numérique)

Découvrir la revue

Citer ce document

Pichette, J.-P. \& d'Entremont, C. (2009). L'édition des contes de tradition orale. Pour qui ? Comment ? Rabaska, 7, 135-137. https://doi.org/10.7202/038345ar d'utilisation que vous pouvez consulter en ligne. 


\section{L'édition des contes de tradition orale. Pour qui ? Comment ?}

Jean-Pierre Pichette et Carmen d’Entremont

Université Sainte-Anne

\section{Journées internationales d'étude}

Du 23 au 26 octobre 2008, la Chaire de recherche en oralité des francophonies minoritaires (COFRAM) et le Groupe de recherche en études acadiennes (GRÉA) de l'Université Sainte-Anne convoquaient à la Pointe-de-l'Église de nouvelles journées internationales d'étude autour du thème « L'Édition des contes de tradition orale. Pour qui ? Comment? ». Organisées en partenariat avec la Chaire de recherche McCain en ethnologie acadienne (CREMEA, Université de Moncton), le département de Folklore et ethnologie de l'Amérique française (Dfeus, Université de Sudbury), le Centre interuniversitaire de recherche en littérature et en culture québécoises (CRILCQ, Université Laval) et le Centre de recherche bretonne et celtique (CRBC, Université de Bretagne occidentale), ces discussions s'inscrivaient dans la réflexion du projet ÉCLORE qui mènera à l'édition d'un corpus de littérature orale. Elles furent l'occasion d'honorer la mémoire de Marius Barbeau (1883-1969) en cette année du $125^{\mathrm{e}}$ anniversaire de sa naissance, lui dont les nombreuses publications marquèrent le passage de l'oral à l'écrit au Canada français.

S'agissant de l'élaboration d'un modèle d'édition de narrations populaires, nous n'avions convié que des professionnels reconnus en matière de tradition orale, ethnologues pour la plupart, mais aussi spécialistes sensibles aux propriétés linguistiques, littéraires, historiques, géographiques, pédagogiques ou psychologiques que véhiculent les récits populaires, et qui se sont frottés par leurs travaux au problème du passage de l'oral à l'écrit, notamment par l'analyse, la comparaison et l'édition de récits des $\mathrm{XIX}^{\mathrm{e}}$ et $\mathrm{XX}^{\mathrm{e}}$ siècles. De plus, chacun d'eux représentait une région de la francophonie nord-américaine (Ontario, Québec, Acadie, États-Unis) et européenne (Bretagne, Occitanie, Île-de-France) afin de procurer une vision plus entière des problèmes que pose l'écriture de l'oralité et, partant, d'autoriser éventuellement le transfert du modèle à la plupart des contextes de la francophonie internationale, voire son adaptation à d'autres langues. 
Avec le chercheur principal (J.-P. Pichette) et son assistante (Carmen d'Entremont), et sous la présidence de Jean-Louis Robichaud, du GrÉA, le séminaire réunissait ses partenaires : Marcel Bénéteau (Sudbury), Aurélien Boivin (Québec), Ronald Labelle (Moncton) et Fañch Postic (Brest) ; et ses collaborateurs : Barry Ancelet (Lafayette), Nicole Belmont (Paris), Bertrand Bergeron (Alma), Josiane Bru (Toulouse), Patricia Casteret-Heiniger (Pau) et Amanda LaFleur (Lafayette). Chacun livra son point de vue sur la méthode de transcription et la composition du recueil de contes d'après son expérience particulière des difficultés rencontrées et des meilleures solutions trouvées. Quelques observateurs se joignirent également à nos invités et enrichirent les échanges.

\section{Axes de discussion}

Les axes de discussion se déployèrent en quatre séances de travail examinant les questions fondamentales qu'ils soulèvent : 1 - Première séance : Transcrire pour qui ? Qui sont les destinataires de la collection de contes et de récits populaires projetée ? Les ethnologues, les linguistes, les littéraires, les enseignants, le grand public ? Une même formule peut-elle vraiment satisfaire ces divers lecteurs ? 2 - Deuxième séance : Transcrire, traduire ou récrire? Comment passer à l'écrit des contes oraux ? Est-il possible de respecter fidèlement la langue du conteur et de produire une transcription accessible au lecteur? 3 - Troisième séance : Qu'advient-il des contes en l'absence de l'oral? Comment traiter les documents manuscrits et les témoignages littéraires qui sont incomplets, résumés ou retouchés ? En plus des textes, de quoi devrait se composer un recueil ou une collection de contes? Quels sont les éléments qu'il est toujours nécessaire d'y présenter? Quels sont ceux qu'il est souhaitable d'ajouter s'ils sont disponibles ? 4 - Quatrième séance : cette séance, tenue en privé sans les observateurs, fit la synthèse des points de convergence et formula les questions que le suivi aura pour tâche de régler.

\section{Résultats}

L'atelier posait deux questions fondamentales concernant «L'édition des contes de tradition orale ». La première question « pour qui ? » cherchait à démêler le problème qui a toujours constitué l'obstacle majeur et qui faisait que chaque auteur-transcripteur-éditeur avait sa méthode particulière que soutenait un argumentaire que lui seul connaissait. Le premier résultat palpable de cette rencontre fut de mettre en commun ces argumentaires particuliers et de réfléchir sur le public lecteur visé. 
De la réponse concertée à la première question, découla la réponse à la deuxième « comment ? ». Ici, à partir d'un protocole élaboré par le chercheur principal il y a trente ans et d'une anthologie réunissant d'autres méthodes élaborées par la suite, chacun des participants, à tour de rôle, puis des autres invités à la période de questions, y alla de son expérience et de ses propositions. Ainsi, on a pu discerner entre l'idéal et le possible pour établir une norme commune qui tienne compte des expertises de la francophonie internationale, nord-américaine (Ontario, Québec, Acadie, Louisiane et États-Unis) et européenne (Bretagne, Occitanie, Île-de-France).

La discussion finale réussit à faire le point sur les éléments de convergence et les défis qui se posent aux éditeurs de textes populaires et mena à une entente sur les principes de base d'un protocole de transcription et sur les étapes de rédaction du rapport.

C'était là une première. D'où l'importance de publier la réflexion de ces journées d'étude avec les règles qui ont donné lieu à un protocole renouvelé de transcription des récits pour leur édition, mais aussi, concernant le contenu du recueil de contes, d'un prototype idéal. Ce protocole a fait l'objet d'un suivi après les rencontres d'octobre 2008 ; il est en cours de révision et paraîtra avec les actes de cet atelier à l'automne 2009 ou à l'hiver 2010.

\section{Projet d'édition ÉClORE}

Cet atelier était le prélude au vaste projet d'édition qui a donné naissance à la chaire COFRAM, c'est-à-dire l'Établissement d'un corpus de littérature orale (ÉCLORE). Un volet préliminaire de ce programme est déjà en cours, mais, grâce à cette rencontre, la solution du problème de l'édition nous paraît maintenant satisfaisante. La réflexion qui a eu lieu lors de cet atelier, comme aussi le protocole dégagé, servira de balise et de guide pratique pour les étapes à venir. Commandité par le Conseil de recherches en sciences humaines du Canada (Ottawa) et le Consulat général de France dans les provinces atlantiques (Moncton), l'événement était aussi parrainé par le Festival de la parole de la baie Sainte-Marie (Pointe-de-l’Église). 